\title{
Parenting Styles and Disordered Eating Among Youths: A Rapid Scoping Review
}

\author{
Chloe Hampshire, Bérénice Mahoney and Sarah K. Davis*
}

School of Psychology, University of Worcester, Worcester, United Kingdom

Youth is a critical period in the development of maladaptive eating behaviors. Previous systematic reviews suggest the etiological significance of parent-child relationships for the onset of disordered eating in youth, but less is known about the role of parenting styles. This rapid scoping review aimed to identify whether research supports the role of parenting styles in the development of disordered eating symptoms among youths. Sixteen studies, retrieved from three databases (PsycArticles, Psyclnfo, and BASE), met the inclusion criteria: original studies, published in English, examined the effect of parenting styles (authoritative or neglectful) on cognitive (drives for thinness and body dissatisfaction) and behavioral (weight control behaviors) disordered eating outcomes,

OPEN ACCESS

Edited by:

Ana Rosa Sepúlveda, Autonomous University of Madrid,

Spain

Reviewed by:

Andrea Sabrina Hartmann University of Konstanz, Germany

Chiara Tosi,

Autonomous University of Madrid,

Spain

*Correspondence:

Sarah K. Davis

sarah.davis@worc.ac.uk

Specialty section:

This article was submitted to Personality and Social Psychology, a section of the journal Frontiers in Psychology

Received: 26 October 2021 Accepted: 21 December 2021 Published: 27 January 2022

Citation:

Hampshire C, Mahoney B and Davis SK (2022) Parenting Styles and Disordered Eating Among Youths: A Rapid Scoping Review.

Front. Psychol. 12:802567. doi: 10.3389/fpsyg.2021.802567 among young people up to 18 years of age. Studies supported an association between various youth disordered eating symptoms such as unhealthy weight control behaviors, and experiences of adverse parenting styles characterized by high levels of control and low levels of responsiveness. Associations between adverse parenting styles and youth disordered eating were frequently indirect and differed depending on the sex of the parent and offspring. Synthesis of findings was limited due to variation in the operationalization and measurement of parenting styles, family context and disordered eating across studies. Longitudinal and standardized research is required to better understand the dynamic associations between parenting styles and youth disordered eating. Implications for family-based care in clinical practice are discussed.

Keywords: youth, adolescents, parenting styles, authoritative, neglectful, disordered eating, family context

\section{INTRODUCTION}

Disordered eating (DE) refers to maladaptive attitudes, behaviors, and cognitions related to eating and weight (Stice et al., 2010), and has been broadly applied to both clinical (Deas et al., 2011) and subclinical (National Health Service, 2018) populations. In the present review, DE encapsulates a subthreshold presentation of eating disorder (ED) symptomology in terms of severity and frequency (e.g., National Health Service, 2018). EDs - including anorexia nervosa, bulimia nervosa, binge eating disorder, other specified feeding and/or eating disorder, and avoidant/restrictive food intake disorder - are clinically diagnosed and involve the maladaptive use of food as a coping mechanism (National Health Service, 2021). DE may predict an ED later in life (Stice and Van Ryzin, 2019), with longitudinal research tracking the progression of subclinical symptoms into severe symptomology (Herle et al., 2020). Given that DE is a frequent antecedent of an ED diagnosis (for overview, see McClelland et al., 2020), evaluating the onset of DE is important for 
understanding the clinical course of EDs and, additionally, the improvement of health among subclinical populations. Further, youth (here defined as young people up to 18 years of age, e.g., Grogan et al., 2020) may be a critical period for DE onset. DE frequently manifests in teenage years (Elmasry and Khali, 2018), though children as young as eight have presented DE (Yilmaz Kafali et al., 2020). Engagement in weight control behaviours (WCB) is common in young people (estimated prevalence rate of $44.4 \%$, Solmi et al., 2021) and occurs more frequently than a clinical diagnosis of an ED (Elmasry and Khali, 2018).

The influence of the parent-child relationship on offspring DE has been explored (Botta and Dumlao, 2009). One component of the parent-child relationship is parenting styles - the typical attitudes held, and behaviors exerted, by those occupying a parenting role (Baumrind, 1971, 1991). Whilst domain-specific behaviors are indicative of parenting practices (such as pressure to eat or weight criticism), parenting styles are characterized by the childrearing attitudes and behaviors presenting across a range of parenting contexts (Darling and Steinberg, 1993; Power, 2013). Parenting styles have been categorized into four typologies comprising of dimensional constructs of responsiveness and demandingness (Maccoby, 1994; Gorostiaga et al., 2019): authoritative, authoritarian, permissive, and neglectful (Baumrind, 1991). Putatively considered adverse parenting styles include authoritarian (exhibiting high demandingness and low responsiveness), permissive (exhibiting low demandingness and high responsiveness), and neglectful (exhibiting low demandingness and low responsiveness) typologies (Baumrind, 1991). Parenting styles demonstrating increased parental indifference (exhibiting low responsiveness to offspring needs) are associated with a range of ED diagnoses, including anorexia and bulimia nervosa (Grogan et al., 2020). Moreover, these "unfavorable" parenting styles have been etiologically implicated in later stages of illness (Stice and Van Ryzin, 2019). It is therefore critical to understand this association in earlier illness- and life-stages before subclinical symptoms develop into serious mental illness.

Parenting styles may be viewed as a process through which attachment with caregivers is established and maintained across development (Neppl et al., 2019). Parents' fostering of a nurturing bond with infants is critical; optimal internal working models (predictively guiding future psychosocial functioning) are established by an early secure bond and are necessary for adaptive development (Bowlby, 1977). To this end, there is evidence to suggest insecure attachment styles are both directly (Jewell et al., 2016) and also indirectly (Gugliandolo et al., 2020), associated with youth DE. Indirect associations suggests theories of attachment offer an important theoretical lens for understanding parental risk pathways to the onset of DE in youth.

Within such risk pathways, youths' experience of adverse parenting styles may be conceptualized as a non-abuse adverse life experience (ALE). Non-abuse ALEs refer to adverse experiences excluding abuse (e.g., sexual or physical) (Grogan et al., 2020), and are characterized by prolonged exposure wherein associated (frequently detrimental) impacts accumulate over time (Cavelzani et al., 2018). Youths' experience of adverse parenting styles may be conceptualized as a non-abuse ALE given that parenting styles remain relatively stable throughout offspring youth development (Zhang et al., 2017), and have been associated with a variety of offspring psychopathological outcomes, including an ED in youth (Erriu et al., 2020) and adulthood (Grogan et al., 2020).

Although some studies have examined the impact of adverse parenting styles on DE symptoms in youth, the results lack consistency. Though demanding (McEwen and Flouri, 2009) and unaccepting (Kerr et al., 2021) parenting styles have been associated with youth $\mathrm{DE}$, non-significant associations have also been reported between paternal (Zubatsky et al., 2015) and maternal (Berge et al., 2014) parenting styles and adolescent DE. As the parent-child relationship is dynamic throughout youth development - with parental influence decreasing in salience relative to other social influences (e.g., Branje, 2018) - parenting styles may not be as influential on offspring outcomes as youths mature. Empirical inconsistencies may also arise from variation in the way that parenting styles and DE have been operationalized and measured. For instance, studies inconsistently capture collective parental contributions within co-parenting contexts (Kuppens and Ceulemans, 2019) with some studies converging maternal and paternal parenting styles in measurement (e.g., Rozenblat et al., 2017). Further, unvalidated tools have been implemented (e.g., Zubatsky et al., 2015). Therefore, as the literature on parenting styles is patterned by conceptual, methodological, and empirical inconsistency, it is necessary to conduct a review (Aromataris and Pearson, 2014).

Previous systematic reviews have evaluated the etiological significance of the parent-child relationship for the onset for both clinical and subclinical maladaptive eating behaviors in youth. Insecure attachment styles (Jewell et al., 2016) and dysfunctional family systems (Langdon-Daly and Serpell, 2017) have been associated with DE and ED symptom presentation among teenagers, respectively. However, to date, a focused systematic search has not been conducted for parenting styles and maladaptive eating behaviors among youth samples. For the present rapid scoping review, a symptom-based approach is undertaken due to (1) the relevance of DE to youth maladaptive eating behaviors (Elmasry and Khali, 2018; Yilmaz Kafali et al., 2020) and (2) the illness progression of DE into an ED (Stice and Van Ryzin, 2019). In sum, the current review aims to establish to what extent the evidence suggests that youths with experience of adverse parenting styles present with DE symptoms by systematically reviewing and methodologically evaluating all relevant literature in the field.

\section{METHODS}

In this rapid scoping review, a systematic search of the literature was conducted (Figure 1). SD \& $\mathrm{CH}$ decided on the scope and focus of the review and selection of search terms and inclusion criteria. Given that the present study is not a full systematic review, a single-reviewer coding and analysis of studies was deemed appropriate and was undertaken by $\mathrm{CH}$ in discussion with SD, as per Gartlehner et al. (2020). 


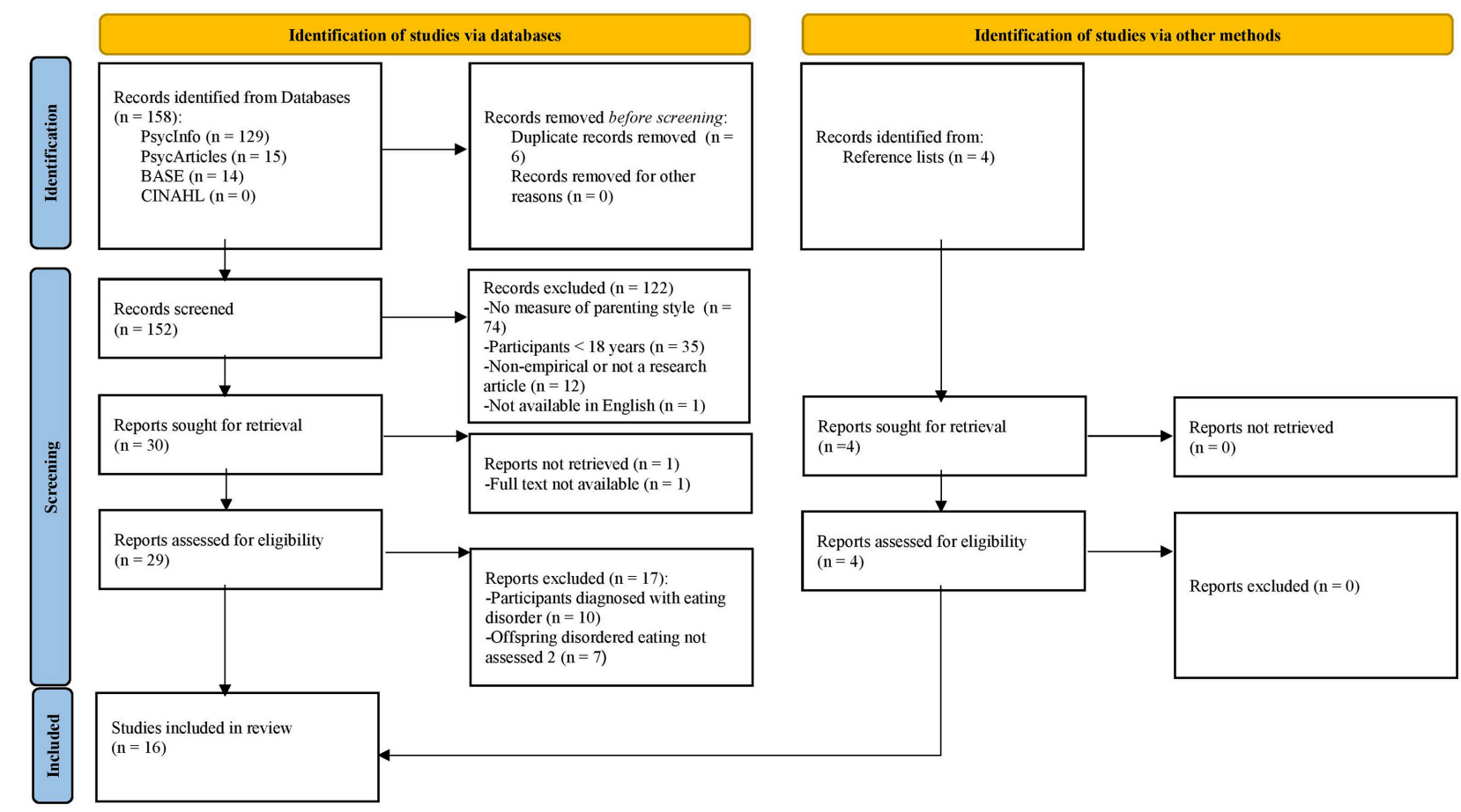

FIGURE 1 | PRISMA 2020 flow diagram for new systematic reviews which included searches of databases and other sources (Page et al., 2021).

\section{Search Strategy}

PsycArticles, PsycInfo, and CINAHL were searched in March 2021 to gather primary published studies. The BASE database was also searched for gray literature for additional relevant sources. Parenting style and teenage or childhood (with synonyms) were used as search terms, combined with the following (including their abbreviations, synonyms, and derivatives): disordered eating and maladaptive eating. Full search strings are included in the Appendix. Manual searches of reference lists of relevant articles were conducted to source potential studies not included in original database searches.

\section{Inclusion and Exclusion Criteria}

Included studies met the following requirements: (1) included a youth sample (up to 18 years of age, e.g., Grogan et al., 2020); (2) incorporated a measure of parenting style; (3) participants had not been diagnosed with an ED; (4) were empirical and not a theoretical paper or meta-analysis; (5) quantitative; (6) published after 1980, corresponding with the publication of the DSM-III (e.g., Grogan et al., 2020); and (7) available in English.

Further to criteria (2); studies solely measuring parenting behaviors relating to food or exercise, such as food restriction (e.g., Loth et al., 2014), were excluded from the review as these are indicative of domain-specific parenting practices (e.g., Darling and Steinberg, 1993) and not of parenting styles presenting across a range of situations (e.g., Baumrind, 1971). Furthermore, (5); as a systematic search has not been conducted for parenting styles and youth populations in the ED field, there were no restrictions on the type of study design included in order to capture all relevant research.

\section{Data Extraction and Synthesis}

One hundred and fifty eight studies were identified from initial database searches. Following the removal of duplicates and screening, 122 studies were excluded, primarily for not including a measure of parenting style or for using an adult sample. Fulltexts of the remaining 34 studies were evaluated, yielding 16 eligible studies for inclusion. Studies were excluded for using participants with an ED diagnosis or not assessing offspring DE.

Data from selected studies were extracted by $\mathrm{CH}$ and included studies' author; year; country; design; sample characteristics; measure of parenting style and DE; parents assessed; risk of bias; and findings (Table 1). Given the heterogenous selection of DE outcomes and measures of parenting styles, a narrative synthesis was deemed the most appropriate form of analysis.

\section{Quality and Risk of Bias Assessment}

A modified version of the Effective Public Health Practice Project (EPHPP) Quality Assessment Tool for Quantitative Studies (Effective Public Health Practice and Project, 1998) was used to evaluate study quality and risk of bias. The following main domains were assessed: selection bias; study design; confounders; data collection methods; withdrawals and drop-outs; and appropriacy of analysis ("blinding" and "intervention integrity" were not relevant for the scope of this review). Included studies' final score following quality appraisal were graded as "weak," "moderate," or "strong" (see Table 1). Studies with domains with 
TABLE 1 | Characteristics of cross-sectional and longitudinal studies analyzing the effect of parenting styles on youth presentation of disordered eating.

\begin{tabular}{|c|c|c|c|c|c|c|c|c|}
\hline References & Study design & $\begin{array}{l}\text { Country of } \\
\text { origin }\end{array}$ & Sample characteristics & $\begin{array}{l}\text { Parenting style } \\
\text { measure }\end{array}$ & Parents assessed & $\begin{array}{l}\text { Disordered eating } \\
\text { measure }\end{array}$ & Relevant findings & $\begin{array}{l}\text { Quality } \\
\text { appraisal }\end{array}$ \\
\hline $\begin{array}{l}\text { Berge et al., } \\
2014\end{array}$ & Cross-sectional & United States & $\begin{array}{l}N=2,793 \text { (1,307 females) } \\
M=14.4 \text { vears, S.D. }=2.0 ; \\
\text { range }=11-19\end{array}$ & $\begin{array}{l}\text { Family assessment } \\
\text { device }\end{array}$ & Mother and father & $\begin{array}{l}\text { Eating and activity in } \\
\text { teens (EAT-26) }\end{array}$ & 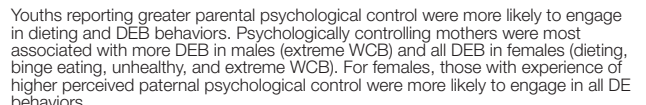 & Moderate \\
\hline $\begin{array}{l}\text { Blodgett } \\
\text { Salafilia et al., } \\
2009\end{array}$ & $\begin{array}{l}\text { Longitudinal } \\
\text { attrition } N / A\end{array}$ & United States & $\begin{array}{l}N=131(73 \text { females }) M=11.65 \text { years; } \\
\text { S.D. }=0.51 \text {; range }=11-13 \text { years }\end{array}$ & $\begin{array}{l}\text { Psychological control } \\
\text { scale-youth } \\
\text { sel-fepont }\end{array}$ & Mother & $\begin{array}{l}\text { Eating Disorder } \\
\text { Inventory (EDI-1) }\end{array}$ & 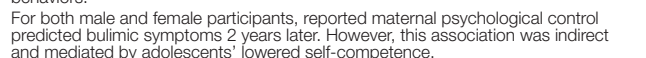 & Weak \\
\hline $\begin{array}{l}\text { Blodgett } \\
\text { Salafia et al., } \\
2007\end{array}$ & $\begin{array}{l}\text { Longitudinal } \\
\text { attrition } N / A\end{array}$ & United States & $\begin{array}{l}N=73(73 \text { female) }) M=11.59 \text { years; } \\
\text { S.D. }=0.52 \text {; range }=11-13 \text { years }\end{array}$ & $\begin{array}{l}\text { Psychological control } \\
\text { scale youth report } \\
\text { democract scale } \\
\text { parental ynowledge } \\
\text { scale }\end{array}$ & Mother & $\begin{array}{l}\text { Eating Disorder } \\
\text { Inventory (EDl-1) }\end{array}$ & 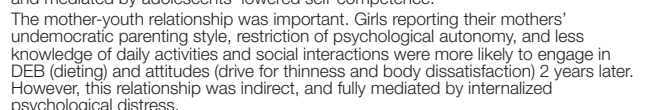 & Moderate \\
\hline $\begin{array}{l}\text { Fonseca } \\
\text { et al., } 2002\end{array}$ & Cross-sectional & Portugal & $\begin{array}{l}N=9,042(4,625 \text { female }) \\
\text { Range }=12-18 \text { years }\end{array}$ & $\begin{array}{l}\text { Voice of Connecticut } \\
\text { youth }\end{array}$ & Converged & $\begin{array}{l}\text { Voice of Connecticut } \\
\text { youth }\end{array}$ & 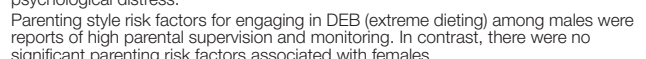 & Weak \\
\hline $\begin{array}{l}\text { Furnham and } \\
\text { Adam-Saib, } \\
2000\end{array}$ & Cross-sectional & United Kingdom & $\begin{array}{l}N=168 \text { (168 female) } \\
\text { Range }=15-17 \text { years }\end{array}$ & $\begin{array}{l}\text { Parental bonding } \\
\text { instrument }\end{array}$ & Mother and father & $\begin{array}{l}\text { Eating attitidues test } \\
\text { (EAT-26) }\end{array}$ & 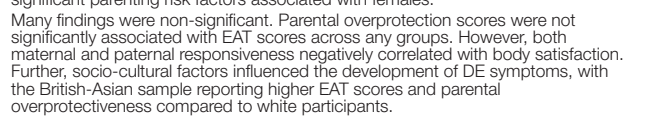 & Weak \\
\hline $\begin{array}{l}\text { Hautala et al., } \\
2011 \text {. }\end{array}$ & $\begin{array}{l}\text { Longitudinal } \\
\text { To } N=N / A \\
\text { T1 } N=1,891 \\
\text { T2 } N=935\end{array}$ & Finland & $\begin{array}{l}N=722(535 \text { female) } M=14.9 \text { years. } \\
\text { Divided into reported eating } \\
\text { disturbance }(n=208) \text {, and } \\
\text { non-symptomatic controls }(n=514)\end{array}$ & $\begin{array}{l}\text { Parental Bonding } \\
\text { Instrument }\end{array}$ & Mother and father & $\begin{array}{l}\text { SCOFF(Finnish } \\
\text { translation) }\end{array}$ & 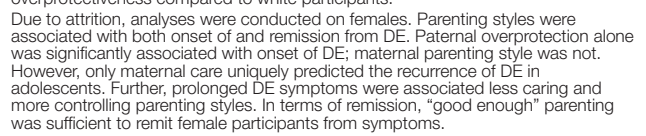 & Moderate \\
\hline $\begin{array}{l}\text { Hochgraf } \\
\text { et al, } 2017\end{array}$ & Cross-sectional & United States & $\begin{array}{l}N=699(351 \text { female) } \\
\text { Range }=11-12 \text { years }\end{array}$ & $\begin{array}{l}\text { Parental Warmth } \\
\text { report }\end{array}$ & Mother and father & $\begin{array}{l}\text { Child's eating habits } \\
\text { and body self-image } \\
\text { scale }\end{array}$ & 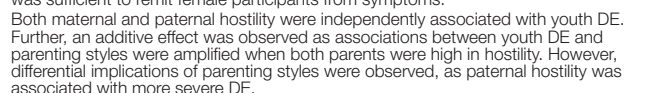 & Weak \\
\hline $\begin{array}{l}\text { Kerr et al., } \\
2021\end{array}$ & $\begin{array}{l}\text { Longitudinal } \\
\text { To } N=4,950 \\
T 1 N=4,950\end{array}$ & United States & $\begin{array}{l}\text { To } N=4,950(2,370 \text { female }) \\
\text { Range }=9-10 \text { years }\end{array}$ & $\begin{array}{l}\text { Children's report of } \\
\text { parent behavior } \\
\text { inventory }\end{array}$ & Mother and father & $\begin{array}{l}\text { Kiddie schedule for } \\
\text { affetive disorders } \\
\text { and Schizophrenia }\end{array}$ & 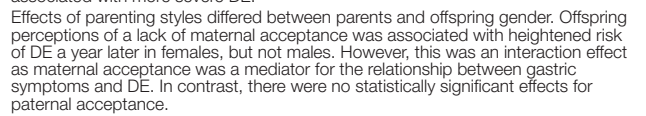 & Moderate \\
\hline $\begin{array}{l}\text { Korotana } \\
\text { et al, } 2018\end{array}$ & $\begin{array}{l}\text { Longitudinal } \\
T 1 N=446 \\
T 1 N=383 \\
T 2 N=352\end{array}$ & Canada & $\begin{array}{l}\text { To } N=446 \text { (466 female) } \\
\text { Range }=11-17 \text { years }\end{array}$ & $\begin{array}{l}\text { Parental } \\
\text { environmental } \\
\text { questionnaire }\end{array}$ & Mother and father & $\begin{array}{l}\text { Minnesota eating } \\
\text { behavior survey }\end{array}$ & 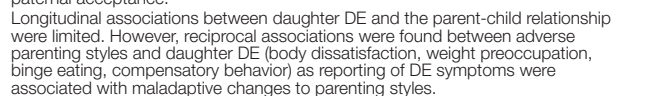 & Strong \\
\hline $\begin{array}{l}\text { Krug et al. } \\
(2014)\end{array}$ & $\begin{array}{l}\text { Longitudinal } \\
\text { Attrition } N / A\end{array}$ & Australia & $\begin{array}{l}N=1,391 \text { ( }(684 \text { female) } \\
M=11.59 \text { years; } S . D \text {. })=0.52 \\
\text { range }=11-13 \text { years) }\end{array}$ & N/A & N/A & $\begin{array}{l}\text { Eating Disorder } \\
\text { Inventory (unspecified) }\end{array}$ & 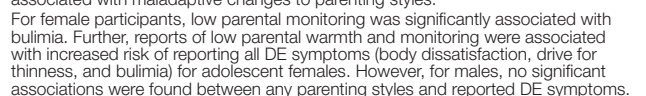 & Moderate \\
\hline $\begin{array}{l}\text { Krug et al., } \\
2016\end{array}$ & $\begin{array}{l}\text { Longitudinal } \\
\text { Attrition } N / A\end{array}$ & Australia & $\begin{array}{l}N=1,300(667 \text { female }) \\
\text { Range }=15-16 \text { years }\end{array}$ & $\begin{array}{l}\text { ATP parenting } \\
\text { practices } \\
\text { questionnaire }\end{array}$ & $\begin{array}{l}1 \text { parent }(95 \% \\
\text { motherr) }\end{array}$ & $\begin{array}{l}\text { Eating Disorder } \\
\text { lnventory (EDI-1) body } \\
\text { dissatisfaction scale }\end{array}$ & 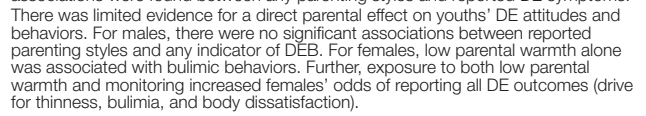 & Moderate \\
\hline $\begin{array}{l}\text { McEwen and } \\
\text { Flouri, } 2009\end{array}$ & Cross-sectional & United Kingdom & $\begin{array}{l}N=203 \text { ( } 125 \text { female) } M=14.04 \text { years; } \\
\text { S.D. }=1.91 ; \text { range }=11-18 \text { years }\end{array}$ & $\begin{array}{l}\text { Parental bonding } \\
\text { instrument parental } \\
\text { control scales }\end{array}$ & Father & $\begin{array}{l}\text { Eating attitudes test } \\
(\text { EAT-26) }\end{array}$ & 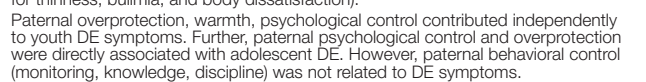 & Strong \\
\hline $\begin{array}{l}\text { Meesters } \\
\text { et al., } 2007\end{array}$ & Cross-sectional & Netherlands & $\begin{array}{l}N=405(224 \text { female) } M=12.5 ; \\
\text { S.D. }=1.5 ; \text { range }=10-16 \text { years }\end{array}$ & $\begin{array}{l}\text { Egna Minnen } \\
\text { Betriftanded } \\
\text { Upofirstran, (Swedish } \\
\text { "My memories of } \\
\text { upboringing") }\end{array}$ & Mother and father & $\begin{array}{l}\text { Children's Eating } \\
\text { Attitudes Test (ChEAT) }\end{array}$ & 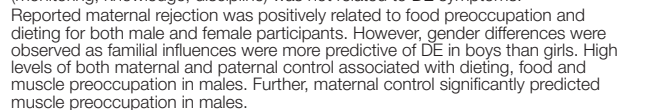 & Moderate \\
\hline $\begin{array}{l}\text { Pearson } \\
\text { et all, } 2009\end{array}$ & Cross-sectional & United Kingdom & $\begin{array}{l}N=328(142 \text { female }) \\
\text { Range }=12-16 \text { years } \\
\text { youngerided inticicipants } \\
M=13.3 \text { years, and older participants } \\
M=15.6 \text { years and }\end{array}$ & $\begin{array}{l}\text { Parenting style } \\
\text { measure made by } \\
\text { Kremers et al. (2003) }\end{array}$ & Converged & $\begin{array}{l}\text { Youth/adolescent } \\
\text { food frequency } \\
\text { questionnaire }\end{array}$ & $\begin{array}{l}\text { No significant interactions between parental involvement or strictness and any } \\
\text { dietary behaviors were found after controlling for gender and age. }\end{array}$ & Strong \\
\hline $\begin{array}{l}\text { Rozenblat } \\
\text { et al., } 2017\end{array}$ & Cross-sectional & Australia & $\begin{array}{l}\text { Study } 1 . N=650 \text { (338 female) } \\
\text { Range }=15-16 \text { years Study } 2 . \\
N=304 \text { (161 female) }\end{array}$ & $\begin{array}{l}\text { Australian } \\
\text { temperament project } \\
\text { parenting practices } \\
\text { scale lowa family } \\
\text { interaction ratiling } \\
\text { scale }\end{array}$ & $\begin{array}{l}1 \text { parent (mainly } \\
\text { mother, no statistic } \\
\text { available) }\end{array}$ & $\begin{array}{l}\text { Eating Disorder } \\
\text { Inventory (EDI-2) }\end{array}$ & 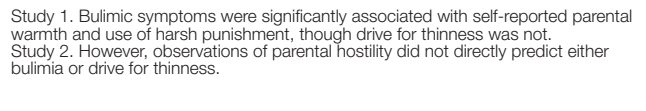 & Weak \\
\hline $\begin{array}{l}\text { Zubatsky } \\
\text { et al, } 2015\end{array}$ & $\begin{array}{l}\text { Longitudinal } \\
\text { attrition } N / A\end{array}$ & Switzerland & $\begin{array}{l}N=2,516(1,386 \text { female) Divided into } \\
\text { middle school, } M=12.8 \text { years; } \\
S . D \text {. }=0.8 \text { at time } 1 \text {, and } \\
M=17.2 \text { years; } S . D=0.6, \text { at time } 2 \\
\text { And high school, } M=15.8 \text { years; } \\
S . D .=0.8 \text { at time } 1 ; M=20.4 ; \\
S . D .=0.8 \text {, at time } 2\end{array}$ & $\begin{array}{l}\text { Created for study } \\
\text { (assessing } \\
\text { authoritative, } \\
\text { authoritarian, } \\
\text { permissive, and } \\
\text { neglectful parenting) }\end{array}$ & Mother and father & $\begin{array}{l}\text { Created for study } \\
\text { (binge eating, WCBs, } \\
\text { extreme WCBs) }\end{array}$ & 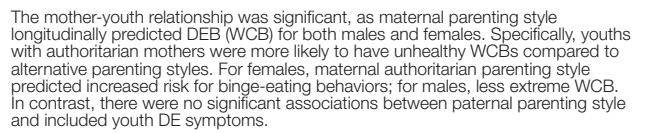 & Moderate \\
\hline
\end{tabular}


$\geq 2,1$, and 0 weak ratings were considered to demonstrate "weak," "moderate," and "strong" risk of bias, respectively.

\section{RESULTS}

Eight studies were cross-sectional and eight were longitudinal (Table 1). All studies used community samples to assess DE, though one also included non-symptomatic controls (Hautala et al., 2011). Included studies were predominantly conducted in English-speaking countries, though studies also originated from Portugal (Fonseca et al., 2002), Netherlands (Meesters et al., 2007), Finland (Hautala et al., 2011) and Switzerland (Zubatsky et al., 2015). Overall, studies inconsistently reported the psychometric properties of tools used, with only 6 studies providing information on both parenting style and DE measures (Blodgett Salafia et al., 2009; Hautala et al., 2011; Berge et al., 2014; Hochgraf et al., 2017; Rozenblat et al., 2017; Korotana et al., 2018).

Parenting styles were assessed via offspring self-report and utilized a range of tools. Parental control scales were implemented in three studies, including the Psychological Control Scale Youth Report (Blodgett Salafia et al., 2007, 2009). The Parental Bonding Instrument (PBI) was implemented in a further three studies among samples including youths younger than the recommended 16 years of age (Furnham and AdamSaib, 2000; McEwen and Flouri, 2009; Hautala et al., 2011).

DE outcomes were measured using self-report tools with the exception of one study, where parents reported on offspring DE using the Kiddie Schedule for Affective Disorders and Schizophrenia (Kerr et al., 2021). Of the rest, five studies used versions of the Eating Disorder Inventory (EDI-1 and -2) and two implemented the Eating Attitudes Test (EAT-26), with one study using a modified version for children (ChEAT, Meesters et al., 2007). The remaining six studies used various alternative tools.

Included studies used a range of statistical analysis techniques, though this information was missing in one study (Krug et al., 2014). Regression models were frequently used, with logistic, linear, and stepwise techniques used by four, two and one studies, respectively. Of the rest, four studies used structural equation modeling, of which one study utilized cross-lagged analyses (Korotana et al., 2018). Notably, structural equation modeling identified associations between parenting styles and youth DE were mediated by offspring emotional reactivity (Hochgraf et al., 2017), psychological distress (Blodgett Salafia et al., 2007) and lowered self-competence (Blodgett Salafia et al., 2009). The remaining four studies used various alternative analyses.

Overall, the divergence in the methodologies, measures and reporting in included studies limited the summarization and synthesis of findings within this scoping review.

\section{Cross-Sectional Studies}

Both paternal (McEwen and Flouri, 2009) and maternal (Meesters et al., 2007) overprotection were correlated with DE food preoccupation. Further, youths who were exposed to controlling parenting styles presented with various DE symptoms, such as internalized muscle preoccupation (Meesters et al., 2007) and externalizing extreme WCBs (Berge et al., 2014). McEwen and Flouri (2009) assessed associations between paternal parenting styles and self-reported DE with additional predictor variables. Paternal psychological control and overprotection were directly associated with all selected indicators of DE behaviors for youths (McEwen and Flouri, 2009). However, conflicting evidence was found as no significant associations were found between any parenting styles and youth dietary behaviors after controlling for a number of potential confounders (Pearson et al., 2009).

\section{Longitudinal Studies}

Similar to the cross-sectional results, adverse parenting presenting varying levels of responsiveness and demandingness was associated with a substantial range of operationalizations of DE. High levels of parental control predicted unhealthy WCBs (Zubatsky et al., 2015) and body dissatisfaction (Blodgett Salafia et al., 2007). Additionally, unresponsive parenting styles (exhibiting low behavioral monitoring) predicted various DE among daughters, such as body dissatisfaction (Blodgett Salafia et al., 2007) and bulimic behaviors (Krug et al., 2014). However, findings were inconsistent as many studies reported non-significant results for males which were not present for their female counterparts (Krug et al., 2014, 2016; Zubatsky et al., 2015; Kerr et al., 2021). Further, some studies compared longitudinal measurements of parental responsiveness and demandingness using logistic regression analyses (Hautala et al., 2011; Krug et al., 2016). Combined (Krug et al., 2016) and prolonged (Hautala et al., 2011) exposure to low parental warmth and high control predicted increased odds of, and more severe, DE. Two studies also aggregated findings at multiple time points throughout the study (Hautala et al., 2011; Korotana et al., 2018). Associations between parenting style and youths' DE were dynamic: crosslagged analyses suggested they were reciprocal (Korotana et al., 2018) and differed with age as associations were stronger in early teens relative to later teenage years (Hautala et al., 2011). Finally, the quality of included studies was restricted by a failure to report attrition rates, with only three studies including this information (Hautala et al., 2011; Korotana et al., 2018; Kerr et al., 2021).

\section{DISCUSSION}

The present review focused on associations between parenting styles and DE presentation in youth. Most studies provided preliminary support for an association between symptoms of DE and experience of various adverse parenting styles (e.g., McEwen and Flouri, 2009; Kerr et al., 2021), although some studies did not (e.g., Fonseca et al., 2002; Korotana et al., 2018). This resonates with previous work that has identified an association between DE beyond youth and other forms of non-abusive ALE present in family contexts, such as family dynamics (Mousoulidou et al., 2019). However, overall, review findings lacked consistency and stability, and associations were often indirect.

A number of characteristics of adverse parenting styles were associated with youth DE. Parenting styles that were perceived as controlling were associated with a range of youth DE outcomes (Meesters et al., 2007; McEwen and Flouri, 2009; Berge et al., 2014). Specifically, paternal overprotection correlated with (McEwen and Flouri, 2009) and increased 
risk of Hautala et al. (2011) DE symptoms. In addition, parental warmth was salient to youth DE, with exposure to low levels associated with a range of DE symptoms (Krug et al., 2014), including bulimia outcomes (Krug et al., 2016).

However, many studies found indirect effects of adverse parenting styles on DE; only one study established a direct association between high paternal demandingness, low responsiveness and DE (McEwen and Flouri, 2009). Associations between adverse parenting styles were mediated by offspring psychological distress (Blodgett Salafia et al., 2007), lowered self-competence (Blodgett Salafia et al., 2009) and emotional reactivity (Hochgraf et al., 2017), thus indicating the affective functioning of young people is an important facilitatory mechanism of DE onset. Attachment theory may thus be useful as a model to explain indirect effects identified in this scoping review, with parenting styles distally influencing youth eating pathology through correlates of the attachment system, such as offspring emotional competency (Laible, 2007) and self-competence (de Minzi, 2010).

Furthermore, longitudinal studies found interactions between parenting styles and DE varied throughout youth (Hautala et al., 2011; Korotana et al., 2018). The teenage years are a transitionary period for the parent-child relationship as parental influence decreases in salience relative to other social influences, including peers (Branje, 2018). It is likely the significance of mediating factors increases as offspring age due to changes in the parent-child relationship (Albarello et al., 2018), and contradictory findings within the review may be attributed to differences in sample age ranges. In sum, longitudinal studies implementing transactional models of parental styles better capture the development of DE across youth life-stages.

\section{Methodological Divergence}

Although many studies assessed and aggregated maternal and paternal parenting styles, some studies converged parental contributions into a unitary construct of parenting in measurement (Pearson et al., 2009) and analysis (Rozenblat et al., 2017). However, the concept of parenting styles has been clarified to acknowledge the coexistence of discrete styles within joint parenting contexts, such as co-parent households (Kuppens and Ceulemans, 2019). Notably, some studies used the marital status of youths' biological parents as inclusion criteria (e.g., Blodgett Salafia et al., 2007). Aggregated evidence from the present review indicate maternal and paternal parenting styles from the same household can differ (Zubatsky et al., 2015) and additively interact (Krug et al., 2014, 2016). Therefore, parents' discrete styles are not consistently captured in convergent concepts of parenting, meaning the utility of current findings is restricted.

A significant differentiator of study quality was researchers' consideration of the confounding effects of co-variates of parenting style. Higher quality studies situated parenting styles in the broader family context, for example collecting selfreported parental marital status (Krug et al., 2016) and number of siblings (Pearson et al., 2009), which has been implicated in modifying parenting style (Fan and Chen, 2020). However, research inconsistently assessed covariates and thus current data were insufficient to interpret meaningful patterns of findings within the review. Additionally, assessments of DE frequently measured drives for thinness and bulimia and body dissatisfaction, though researchers selected various tools to do so. Higher quality studies generally implemented well validated measures, such as the EDI-1 and EAT-26. However, some studies compiled (Berge et al., 2014) or created new measures by selecting operationalizations of $\mathrm{DE}$ from existing tools, without providing sufficient justification for selection (e.g., Fonseca et al., 2002). Collectively, the included studies introduced heterogeneity into the outcome of interest, thereby reducing the ability to synthesize findings and draw symptom-specific conclusions from youth experiences of parenting styles. Standardization of methods (specifically the measurement of covariates of parenting style, $\mathrm{DE}$ outcome and parenting style) is required to strengthen comparative conclusions drawn from the evidence base.

\section{Limitations}

Only one study implemented a multi-modal procedure to assess parenting style (Rozenblat et al., 2017), thereby most studies in this review relied on an assumed convergence between knowledge and functioning (Baumrind, 2005; Herbers et al., 2017). Critically, observations of parenting style demonstrated low convergence with parental self-report (Rozenblat et al., 2017), suggesting conclusions relating to parenting style and youth DE outcomes may be dependent on the mode of assessment. Future research should implement standardized procedures incorporating multi-modal, aggregated assessments of maternal and paternal parenting styles.

Additionally, directional influences of parenting styles cannot be inferred from reported DE symptoms in cross-sectional studies. Though Baumrind (1991) conceptualizes parental influence on offspring outcomes as unidirectional, parenting style has been reconceptualized as bidirectional (Estlein, 2021) and reciprocal (Pinquart and Gerke, 2019), acknowledging the contribution of the offspring in parent-child interactions. Longitudinal evidence from the review indicates that $\mathrm{DE}$ acts a risk factor for adverse parenting, suggesting that youths' DE symptoms subsequently modify parenting styles (Korotana et al., 2018). Establishing the directionality of associations is critical for the accurate interpretation of findings, and future research must continue to implement this design in order to capture the bidirectional and temporally dynamic nature of associations between parenting styles and youth DE throughout offspring development.

Finally, selection bias may be present as review results were limited to the English language. However, as the majority studies were conducted by English speaking countries, the risk of excluding additional relevant findings is anticipated to be low.

\section{Implications}

Findings from the present review have implications for clinical practice. The prevalence of reported DE symptoms in youth across studies demonstrates the clinical necessity of intervention into maladaptive parenting styles. Clinicians must implement a patient-centered recovery model (e.g., Wetzler et al., 2020) and consider parental influences in the treatment of DE; family level interventions must be offered if appropriate to the young person's experiences of DE (see Lock and Le Grange, 2019 
for overview). From the findings of the present review, interventions into parenting styles characterized by controlling (e.g., Berge et al., 2014), overprotective (e.g., McEwen and Flouri, 2009) and unresponsive (e.g., Krug et al., 2016) parental behaviors are recommended. Such interventions may thus prevent the maintenance of youth DE symptoms. In addition, interventive promotion of "good enough" parenting styles exhibiting age appropriate control and responsiveness are recommended to remit youth from DE (Hautala et al., 2011, p.961). Subsequently, consistent with the conceptualization of maladaptive eating behaviors as a continuum from DE to an ED (Dinkler et al., 2021), targeted interventions into parental pathways of risk for the onset of DE can work to reduce family based risk of development and circumvent the development of subclinical symptoms into an ED (Stice and Van Ryzin, 2019).

\section{CONCLUSION}

This rapid scoping review evaluated 16 studies to assess whether parenting style could be a risk factor for youth

\section{REFERENCES}

Albarello, F., Crocetti, E., and Rubini, M. (2018). I and us: a longitudinal study on the interplay of personal and social identity in adolescence. J. Youth Adolesc. 47, 689-702. doi: 10.1007/s10964-017-0791-4

Aromataris, E., and Pearson, A. (2014). The systematic review: an overview. Am. J. Nurs. 114, 53-58. doi: 10.1097/01.NAJ.0000444496.24228.2C

Baumrind, D. (1971). Current patterns of parental authority. Dev. Psychol. Monogr. 4(1 Pt. 2), 1-103.

Baumrind, D. (1991). "Effective parenting during the early adolescent transition," in Family Transitions. Advances in Family Research Series, eds P. A. Cowan and E. M. Hetherington (Hillsdale: Lawrence Erlbaum Associates, Inc). doi: $10.1017 /$ s0954579420002096

Baumrind, D. (2005). Patterns of parental authority and adolescent autonomy. New Direct. Child Adolesc. Dev. 20, 61-69. doi: 10.1002/cd.128

Berge, J. M., Wall, M., Larson, N., Eisenberg, M. E., and Loth, K. A. (2014). The unique and additive associations of family functioning and parenting practices with disordered eating behaviors in diverse adolescents. J. Behav. Med. 37, 205-217. doi: 10.1007/s10865-012-9478-1

Blodgett Salafia, E. H. B., Gondoli, D. M., Corning, A. F., Bucchianeri, M. M., and Godinez, N. M. (2009). Longitudinal examination of maternal psychological control and adolescents' self-competence as predictors of bulimic symptoms among boys and girls. Int. J. Eat. Dis. 42, 422-428. doi: 10.1002/eat.20626

Blodgett Salafia, E. H., Gondoli, D. M., Corning, A. F., Mcenery, A. M., and Grundy, A. M. (2007). Psychological distress as a mediator of the relation between perceived maternal parenting and normative maladaptive eating among adolescent girls. J. Counsell. Psychol. 54, 434-446. doi: 10.1037/00220167.54.4.434

Botta, R. A., and Dumlao, R. (2009). How do conflict and communication patterns between fathers and daughters contribute to or offset eating disorders? Health Commun. 14, 199-219. doi: 10.1207/S15327027HC1402_3

Bowlby, J. (1977). The making and breaking of affectional bonds: II. Some principles of psycho-therapy. Br. J. Psychiatry 130, 421-431. doi: 10.1192/bjp. 130.5.421

Branje, S. (2018). Development of parent-adolescent relationships: conflict interactions as a mechanism of change. Child Dev. Perspect. 12, 171-176. doi: 10.1111/cdep. 12278

Cavelzani, A. S., Trinchieri, M., Gorio, M. C. F., and Romeo, L. (2018). Microtraumatic experiences and psychotherapeutic treatment. J. Integr. Med. 7, 1-6. doi: $10.18282 /$ jim.v7i1.518

Darling, N., and Steinberg, L. (1993). Parenting style as context: an integrative model. Psychol. Bull. 113, 56-78.
DE. Exposure to demanding, unresponsive, or a combination of these, parenting styles both predicted and correlated with reports of various symptoms of DE. However, included results were often conflicted, and comparative conclusions concerning the influence of adverse parenting styles on youth $\mathrm{DE}$ are limited due to the heterogenous operationalization and measurement of parenting styles, family context, and DE outcomes. Therefore, the present review does not establish a definitive account of the etiological influence of parental style to the onset of $\mathrm{DE}$ in youth. Further research implementing longitudinal and standardized procedures is essential for good quality research into parenting styles.

\section{AUTHOR CONTRIBUTIONS}

$\mathrm{CH}$ : conceptualization, methodology, investigation, and writing - original draft. BM: writing - review and editing. SD: conceptualization and writing - review and editing. All authors contributed to the article and approved the submitted version.

de Minzi, M. C. R. (2010). Gender and cultural patterns of mothers' and fathers' attachment and links with children's self-competence, depression and loneliness in middle and late childhood. Early Child Dev. Care 180, 193-209. doi: 10.1080/ 03004430903415056

Deas, S., Power, K., Collin, P., Yellowlees, A., and Grierson, D. (2011). The relationship between disordered eating, perceived parenting, and perfectionistic schemas. Cogn. Ther. Res. 5, 414-424. doi: 10.1007/S10608-010-9319-X

Dinkler, L., Taylor, M. J., Råstam, M., Hadjikhani, N., Bulik, C. M., Lichtenstein, P., et al. (2021). Association of etiological factors across the extreme end and continuous variation in disordered eating in female Swedish twins. Psychol. Med. 51, 750-760. doi: 10.1017/S0033291719003672

Effective Public Health Practice and Project (1998). Quality Assessment Tool For Quantitative Studies. Hamilton, ON: Effective Public Health Practice.

Elmasry, N., and Khali, D. (2018). Subclinical eating disorders in a sample of secondary school girls and comorbidity with depression in Sharkia Governorate, Egypt. Egypt J. Psychiatry 39:129. doi: 10.4103/1110-1105.243024

Erriu, M., Cimino, S., and Cerniglia, L. (2020). The role of family relationships in eating disorders in adolescents: a narrative review. Behav. Sci. 10:88. doi: 10.3390/BS10040071

Estlein, R. (2021). Parenting as a communication process: integrating interpersonal communication theory and parenting styles conceptualization. J. Family Theory Rev. 13, 21-33. doi: 10.1111/jftr.12407

Fan, J., and Chen, B. (2020). Parenting styles and coparenting in China: the role of parents and children's sibling status. Curr. Psychol. 39, 1505-1512. doi: 10.1007/ s12144-019-00379-7

Fonseca, H., Ireland, M., and Resnick, M. D. (2002). Familial correlates of extreme weight control behaviors among adolescents. Int. J. Eat. Dis. 32, 441-448. doi: 10.1002/eat.10078

Furnham, A., and Adam-Saib, S. (2000). Abnormal eating attitudes and behaviours and perceived parental control: a study of white British and British-Asian school girls. Soc. Psychiatry Epidemiol. 36, 462-470. doi: 10.1007/s001270170025

Gartlehner, G., Affengruber, L., Titscher, V., Noel-Storr, A., Dooley, G., Ballarini, N., et al. (2020). Single-reviewer abstract screening missed 13 percent of relevant studies: a crowd-based, randomized controlled trial. J. Clin. Epidemiol. 121, 20-28. doi: 10.1016/J.JCLINEPI.2020.01.005

Gorostiaga, A., Aliri, J., Balluerka, N., and Lameirinhas, J. (2019). Parenting styles and internalizing symptoms in adolescence: a systematic literature review. Int. J. Environ. Res. Public Health 16, 1-19. doi: 10.3390/ijerph16173192

Grogan, K., MacGarry, D., Bramham, J., Scriven, M., Maher, C., and Fitzgerald, A. (2020). Family-related non-abuse adverse life experiences occurring for adults diagnosed with eating disorders: a systematic review. J. Eat. Dis. 8:36. doi: 10.1186/s40337-020-00311-6 
Gugliandolo, M. C., Costa, S., Cuzzocrea, F., Larcan, R., and Martino, G. (2020). Adolescents and body uneasiness: the contribution of supportive parenting and trait emotional intelligence. J. Child Fam. Stud. 29, 2453-2462. doi: 10.1007/ S10826-020-01779-1/FIGURES/1

Hautala, L., Helenius, H., Karukivi, M., Maunula, A.-M., Nieminen, J., Aromaa, M., et al. (2011). The role of gender, affectivity and parenting in the course of disordered eating: a 4-year prospective case-control study among adolescents. Int. J. Nurs. Stud. 48, 959-972. doi: 10.1016/j.ijnurstu.2011.01.014

Herbers, J. E., Garcia, E. B., and Obradović, J. (2017). Parenting assessed by observation versus parent-report: moderation by parent distress and family socioeconomic status. J. Child Fam. Stud. 26, 3339-3350.

Herle, M., Stavola, B. De, Hübel, C., Abdulkadir, M., Ferreira, D. S., Loos, R. J. F., et al. (2020). A longitudinal study of eating behaviours in childhood and later eating disorder behaviours and diagnoses. Br. J. Psychiatry 216, 113-119.

Hochgraf, A. K., Kahn, R. E., and Kim-Spoon, J. (2017). The moderating role of emotional reactivity in the link between parental hostility and eating disorder symptoms in early adolescence. Eat. Dis. 25, 420-435.

Jewell, T., Collyer, H., Gardner, T., Tchanturia, K., Simic, M., Fonagy, P., et al. (2016). Attachment and mentalization and their association with child and adolescent eating pathology: a systematic review. Int. J. Eat. Dis. 49, 354-373. doi: 10.1002 /eat. 22473

Kerr, K. L., Ralph-Nearman, C., Colaizzi, J. M., DeVille, D. C., Breslin, F. J., Aupperle, R. L., et al. (2021). Gastric symptoms and low perceived maternal warmth are associated with eating disorder symptoms in young adolescent girls. Int. J. Eat. Dis. 54, 1009-1018. doi: 10.1002/eat.23516

Korotana, L. M., von Ranson, K. M., Wilson, S., and Iacono, W. G. (2018). Reciprocal associations between eating pathology and parent-daughter relationships across adolescence: a monozygotic twin differences study. Front. Psychol. 9:914. doi: 10.3389/fpsyg.2018.00914

Kremers, S. P. J., Brug, J., De Vries, H., and Engels, R. C. M. E. (2003). Parenting style and adolescent fruit consumption. Appetite 41, 43-50. doi: 10.1016/S01956663(03)00038-2

Krug, I., King, R. M., Youssef, G. J., Sorabji, A., Wertheim, E. H., Le Grange, D., et al. (2016). The effect of low parental warmth and low monitoring on disordered eating in mid-adolescence: findings from the Australian temperament project. Appetite 105, 232-241. doi: 10.1016/j.appet.2016.05.015

Krug, I., Sorabji, A., King, R., Letcher, P., and Olsson, C. (2014). Does low parental warmth and monitoring predict disordered eating in Australian female and male adolescents? J. Eat. Dis. 2:O29. doi: 10.1186/2050-2974-2-S1-O29

Kuppens, S., and Ceulemans, E. (2019). Parenting styles: a closer look at a wellKnown concept. J. Child Fam. Stud. 28, 168-181. doi: 10.1007/s10826-0181242-X

Laible, D. (2007). Attachment with parents and peers in late adolescence: links with emotional competence and social behavior. Pers. Individ. Diff. 43, 1185-1197. doi: 10.1016/J.PAID.2007.03.010

Langdon-Daly, J., and Serpell, L. (2017). Protective factors against disordered eating in family systems: a systematic review of research. J. Eat. Dis. 5:22. doi: 10.1186/s40337-017-0141-7

Lock, J., and Le Grange, D. (2019). Family-based treatment: where are we and where should we be going to improve recovery in child and adolescent eating disorders. Int. J. Eat. Dis. 52, 481-487. doi: 10.1002/eat.22980

Loth, K. A., Maclehose, R., Bucchianeri, M., Crow, S., and Neumark-Sztainer, D. (2014). Predictors of dieting and disordered eating behaviors from adolescence to young adulthood. J. Adolesc. Health 55, 705-712. doi: 10.1016/j.jadohealth. 2014.04.016

Maccoby, E. E. (1994). The role of parents in the socialization of children: an historical overview. Century Dev. Psychol. 68, 589-615.

McClelland, J., Robinson, L., Potterton, R., Mountford, V., and Schmidt, U. (2020). Symptom trajectories into eating disorders: a systematic review of longitudinal, nonclinical studies in children/adolescents. Eur. Psychiatry 63, 1-11. doi: 10. 1192/J.EURPSY.2020.55

McEwen, C., and Flouri, E. (2009). Fathers' parenting, adverse life events, and adolescents' emotional and eating disorder symptoms: the role of emotion regulation. Eur. Child Adolesc. Psychiatry 18, 206-216. doi: 10.1007/s007870080719-3

Meesters, C., Muris, P., Hoefnagels, C., and van Gemert, M. (2007). Social and family correlates of eating problems and muscle preoccupation in young adolescents. Eat. Behav. 8, 83-90. doi: 10.1016/j.eatbeh.2006.02. 002
Mousoulidou, M., Argyrides, M., and Ioannou, M. (2019). Effects of family dynamics on disordered eating patterns and behaviors: evidence from cyprus. Eur. J. Counsell. Psychol. 8, 64-77. doi: 10.5964/ejcop.v8i1.181

National Health Service (2018). Disordered Eating: Guidelines for Schools and Staff. London: National Health Service.

National Health Service (2021). Overview - Eating Disorders. London: National Health Service.

Neppl, T. K., Wedmore, H., Senia, J. M., Jeon, S., and Diggs, O. (2019). Couple interaction and child social competence: the role of parenting and attachment. Soc. Dev. 28:363. doi: 10.1111/SODE.12339

Page, M. J., McKenzie, J. E., Bossuyt, P. M., Boutron, I., Hoffmann, T. C., Mulrow, C. D., et al. (2021). The PRISMA 2020 statement: an updated guideline for reporting systematic reviews. BMJ 372:71. doi: 10.1136/bmj.n71

Pearson, N., Atkin, A. J., Jh Biddle, S., Gorely, T., and Edwardson, C. (2009). Parenting styles, family structure and adolescent dietary behaviour. Public Health Nutr. 13, 1245-1253. doi: 10.1017/\$1368980009992217

Pinquart, M., and Gerke, D. C. (2019). Associations of parenting styles with selfesteem in children and adolescents: a meta-analysis. J. Child Fam. Stud. 28, 2017-2035. doi: 10.1007/s10826-019-01417-5

Power, T. G. (2013). Parenting dimensions and styles: a brief history and recommendations for future research. Child. Obes. 9, S14-S21. doi: 10.1089/ CHI.2013.0034

Rozenblat, V., Ryan, J., Wertheim, E., King, R., Olsson, C. A., and Krug, I. (2017). Relationships between self-reported and observed parenting behaviour, adolescent disordered eating attitudes and behaviours, and the 5-HTTLPR polymorphism: data from the Australian temperament project. Eur. Eat. Dis. Rev. 25, 381-388. doi: 10.1002/erv.2530

Solmi, F., Helen Sharpe, P., Gage, S. H., Maddock, J., Lewis, G., and Patalay, P. (2021). Changes in the prevalence and correlates of weight-control behaviors and weight perception in adolescents in the UK, 1986-2015. JAMA Pediatr. 175, 267-275. doi: 10.1001/JAMAPEDIATRICS.2020.4746

Stice, E., and Van Ryzin, M. J. (2019). A Prospective test of the temporal sequencing of risk factor emergence in the dual pathway model of eating disorders. J. Abnorm. Psychol. 128, 119-128. doi: 10.1037/abn0000400

Stice, E., Ng, J., and Shaw, H. (2010). Risk factors and prodromal eating pathology. J. Child Psychol. Psychiatry 51, 518-525. doi: 10.1111/J.1469-7610.2010.02212.X

Wetzler, S., Hackmann, C., Peryer, G., Clayman, K., Friedman, D., Saffran, K., et al. (2020). A framework to conceptualize personal recovery from eating disorders: a systematic review and qualitative meta-synthesis of perspectives from individuals with lived experience. Int. J. Eat. Dis. 53, 1188-1203. doi: 10.1002/eat.23260

Yilmaz Kafali, H., Atik Altinok, Y., Ozbaran, B., Ozen, S., Kose, S., Tahillioglu, A., et al. (2020). Exploring emotional dysregulation characteristics and comorbid psychiatric disorders in type 1 diabetic children with disordered eating behavior risk. J. Psychosom. Res. 131:109960. doi: 10.1016/j.jpsychores.2020.109960

Zhang, W., Wei, X., Ji, L., Chen, L., and Deater-Deckard, K. (2017). Reconsidering parenting in Chinese culture: subtypes, stability, and change of maternal parenting style during early adolescence. J. Youth Adolesc. 46, 1117-1136. doi: 10.1007/S10964-017-0664-X

Zubatsky, M., Berge, J., and Neumark-Sztainer, D. (2015). Longitudinal associations between parenting style and adolescent disordered eating behaviors. Eat. Weight Dis. 20, 187-194. doi: 10.1007/s40519-014-0154-z

Conflict of Interest: The authors declare that the research was conducted in the absence of any commercial or financial relationships that could be construed as a potential conflict of interest.

Publisher's Note: All claims expressed in this article are solely those of the authors and do not necessarily represent those of their affiliated organizations, or those of the publisher, the editors and the reviewers. Any product that may be evaluated in this article, or claim that may be made by its manufacturer, is not guaranteed or endorsed by the publisher.

Copyright (C) 2022 Hampshire, Mahoney and Davis. This is an open-access article distributed under the terms of the Creative Commons Attribution License (CC BY). The use, distribution or reproduction in other forums is permitted, provided the original author(s) and the copyright owner(s) are credited and that the original publication in this journal is cited, in accordance with accepted academic practice. No use, distribution or reproduction is permitted which does not comply with these terms. 


\section{APPENDIX}

Search strings:

\section{PsycArticles}

Parenting styles or parenting or parental practices.

Disordered eating or eating pathology or maladaptive eating. Adolescents or teenagers or children or kids or youth.

PsycInfo

Parenting styles or parenting or parental practices.

Disordered eating or maladaptive eating or eating pathology.

Adolescents or teenagers or children or teen or youth.

BASE

Parenting styles or parenting.

Disordered eating or eating pathology or maladaptive eating.

Teenagers or adolescents or children.

\section{CINAHL}

Parenting styles or parenting.

Disordered eating or maladaptive eating or eating pathology. 